\title{
Consistent Scheme for Computation of Reynolds Stress and Turbulent Kinetic Energy Budgets for Energy-Conservative Finite Difference Method*
}

\author{
Hiroya MAMORI** and Koji FUKAGATA** \\ ** Department of Mechanical Engineering, Keio University \\ Hiyoshi 3-14-1, Kohoku-ku, Yokohama 223-8522, Japan \\ E-mail: hiroyamamori@z5.keio.jp
}

\begin{abstract}
A consistent scheme for computation of the Reynolds stress and turbulent kinetic energy budgets is proposed for incompressible turbulent flow simulation using the finite difference method on a nonuniform staggered grid system. The present scheme is derived from the Navier-Stokes equation discretized by using the second-order energyconservative finite differential method. The Reynolds stress and turbulent kinetic energy budgets in a fully developed channel flow computed by using the proposed schemes are compared with those by using the consistent scheme proposed for a uniform grid [Suzuki and Kawamura, Trans. JSME/B, Vol. 60, No. 578 (1994), pp. 3280-3286] and an intuitive scheme. The residuals in the Reynolds normal stress and turbulent kinetic energy budgets computed by using the present scheme and Suzuki-Kawamura scheme are found to be both sufficiently small. We also apply the same idea to the discretization of Reynolds shear stress budget, although the consistency is imperfect, be derived the numerical test shows that the residual is slightly smaller than that computed by the other schemes.
\end{abstract}

Key words : Numerical Scheme, Budget, Reynolds Stress, Turbulent Kinetic Energy, Finite Difference Method.

\section{Introduction}

Owing to the recent development of digital computer, direct numerical simulation (DNS) and large eddy simulation (LES) have become handy tools to investigate the fundamental turbulent flow behavior. Since the pioneering DNSs by Orszag and Patterson ${ }^{(1)}$ for homogeneous isotropic turbulence and by Kim et al. (2) for a fully developed incompressible channel flow, the spectral method had been the sole method to stably and accurately simulate the turbulent flows without introducing upwinding which introduces numerical diffusion. Use of the spectral method, however, is limited to canonical flows. Thus, a stable and non-diffusive (i.e., energy-conservative) finite difference method (FDM) had been explored for a long time.

Although the energy-conservative second-order FDM on a uniform grid had been proposed as early as 1960 's ${ }^{(3),(4)}$, we had to wait for 30 years for such FDM to be extended to more practical numerical configurations (such as higher order FDMs, nonuniform grid, etc.). Morinishi et al. ${ }^{(5)}$ presented a class of energy-conservative FDMs on uniform Cartesian grids including generalization to higher order FDMs. Kajishima ${ }^{(7)}$ (also Ham et al. (6) and Bewley ${ }^{(8)}$ ) extended its second-order version to a nonuniform Cartesian grid. To date, energy-conservative FDM has been extended to, e.g., the cylindrical coordinates ${ }^{(9),(10)}$, arbitrary orthogonal curvilinear coordinates ${ }^{(11)}$ and the low Mach number approximation ${ }^{(12)}$.

Analysis of the transport equations for the Reynolds stress (RS) and the turbulent kinetic energy (TKE) (hereafter referred to as RS budget and TKE budget, respectively) is useful

* Received 5 Feb., 2010 (No. 10-0055) [DOI: 10.1299/jcst.4.64]

Copyright (c) 2010 by JSME 


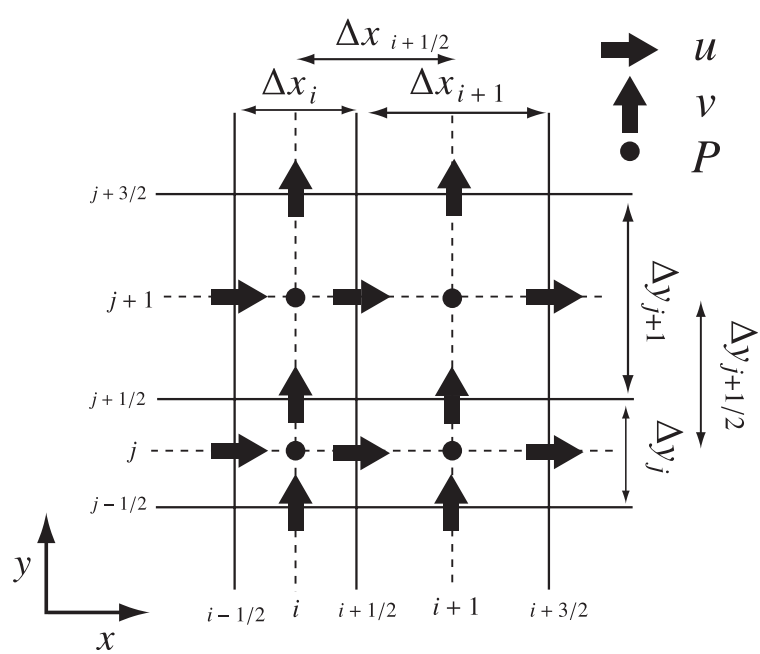

Fig. 1 Nonuniform staggered grid system (two-dimensional view).

for the quantification of different dynamical contributions in turbulence ${ }^{(13)}$. It used be and still is the central issue of turbulence modeling ${ }^{(14),(15)}$; it is also a fundamental tool to clarify the cause of turbulence modification by active control ${ }^{(16),(17)}$. For FDM on the staggered grid, however, it is not straightforward to compute each term in RS and TKE budgets. The quantities composing each term are defined at different locations; thus, interpolation is required. In order to perform a reliable analysis, such interpolation should be consistent with the discretized Navier-Stokes equation.

A consistent scheme for computation of RS and TKE budgets in Cartesian coordinates was proposed by Suzuki and Kawamura ${ }^{(18)}$ (see also Kawamura ${ }^{(19)}$ ). They considered the second-order central FDM on a uniform staggered grid and presented a relevant interpolation scheme for the computation of each term in RS and TKE budgets. Although their scheme had much higher consistency than intuitive interpolation schemes, some issues were left: 1) the turbulent diffusion term was left inconsistent; 2) strictly speaking, their scheme is consistent only on a uniform grid. This is natural because, as introduced above, the general framework of energy-conservative FDM had not been developed by the time Suzuki and Kawamura(18) proposed this scheme. By now, the energy-conservative FDM has become mature: it is now possible to construct a consistent scheme resolving the abovementioned issues.

The objective of this paper is to propose an interpolation scheme used for the computation of RS and TKE budgets, which is consistent with the second-order energy-conservative FDM on a nonuniform staggered grid system ${ }^{(6)-(8)}$. As introduced below, the discretization schemes for the RS and TKE budgets can be derived from the energy-conservative FDM ${ }^{(6)-(8)}$. Although the basic idea is straightforward, to the best of authors' knowledge such schemes are not available in literature, possibly because it needs an extensive amount of calculation in their derivation. The main purpose of this paper is to present such discretization schemes for the RS and TKE budgets on a nonuniform grid. The paper is organized as follows. In Section 2, we review the energy-conservative FDM and the derivation process of RS and TKE budgets in continuous space, which are the bases of the present derivation. In Section 3, we propose the consistent scheme for RS budget; a relevant definition for TKE is also proposed by using the volume average, The proposed scheme is then tested in Section 4 by DNS of a fully developed turbulent channel flow: the RS and TKE budgets computed by using the proposed schemes are compared with those computed by using the existing consistent schemes ${ }^{(18)}$ and the spectral method ${ }^{(20)}$.

\section{Preliminaries}

\subsection{Energy-conservative finite differential method}

Three dimensional Cartesian coordinates are considered. The governing equations are the 
continuity and Navier-Stokes (NS) equations for an incompressible flow. In the continuous space, these equations read

$$
\begin{aligned}
& \frac{\partial u_{n}}{\partial x_{n}}=0 \\
& \frac{\partial u_{n}}{\partial t}=-\frac{\partial u_{k} u_{n}}{\partial x_{k}}-\frac{\partial p}{\partial x_{n}}+\frac{1}{\operatorname{Re}} \frac{\partial^{2} u_{n}}{\partial x_{k} \partial x_{k}},
\end{aligned}
$$

where $\mathrm{Re}$ is the Reynolds number. For notational convenience, $x_{n}$ and $u_{n}$ (for $n=1 \ldots 3$ ) are interexchangably used to denote the directions $(x, y$, and $z)$ and the velocity components $(u, v$, and $w$ ), respectively.

Figure 1 shows the schematic of the nonuniform staggered grid system (two-dimensional view, for simplicity). The velocities and the pressure are defined at the cell surface and the cell center, respectively. The first and second derivatives are discretized, e.g., as

$$
\begin{aligned}
& {\left[\frac{\delta v}{\delta y}\right]_{i, j, k}=\frac{v_{i, j+1 / 2, k}-v_{i, j-1 / 2, k}}{\Delta y_{j}}} \\
& {\left[\frac{\delta^{2} v}{\delta y \delta y}\right]_{i, j+1 / 2, k}=\frac{1}{\Delta y_{j+1 / 2}}\left(\frac{v_{i, j+3 / 2, k}-v_{i, j+1 / 2, k}}{\Delta y_{j+1}}-\frac{v_{i, j+1 / 2, k}-v_{i, j-1 / 2, k}}{\Delta y_{j}}\right)}
\end{aligned}
$$

where the subscripts $i, j$, and $k$ denote the stencils in $x, y$, and $z$ directions, respectively.

Equations (1) and (2) discretized by using the second-order energy-conservative FDM (6) -(8) then read

$$
\begin{aligned}
& \frac{\delta u_{n}}{\delta x_{n}}=0, \\
& \frac{\delta u_{n}}{\delta t}=-\frac{\delta{\widehat{u_{k}}}^{x_{n}}{\overline{u_{n}}}^{x_{k}}}{\delta x_{k}}-\frac{\delta p}{\delta x_{n}}+\frac{1}{\operatorname{Re}} \frac{\delta^{2} u_{n}}{\delta x_{k} \delta x_{k}} .
\end{aligned}
$$

Here, the overbar and $\overparen{(\cdot)}$ denote the arithmetic and volume-flux averages ${ }^{(9)}$, respectively. These are defined, e.g., as

$$
\bar{u}_{i+1 / 2, j+1 / 2, k}^{y}=\frac{u_{i+1 / 2, j+1, k}+u_{i+1 / 2, j, k}}{2},
$$

and

$$
\left\{\begin{aligned}
\hat{u}_{i+1, j, k}^{x} & =\frac{u_{i+1 / 2, j, k}+u_{i-1 / 2, j, k}}{2} \\
\hat{u}_{i+1 / 2, j+1 / 2, k}^{y} & =\frac{\Delta y_{j+1} u_{i+1 / 2, j+1, k}+\Delta y_{j} u_{i+1 / 2, j, k}}{\Delta y_{j+1}+\Delta y_{j}}
\end{aligned}\right.
$$

where the superscripts $(x, y$, and $z$ ) denote the direction of interpolation. The discretized advection term has been verified ${ }^{(6)-(8)}$ to be momentum- and energy-conservative (strictly speaking, squared-value-conservative) given that the discretized continuity equation is satisfied. Thus, the discretized equations (5) and (6) conserve not only the mass and momentum but also the total kinetic energy in the inviscid limit.

\subsection{Reynolds stress and turbulent kinetic energy budgets}

The derivation process of the Reynolds stress budget in the continuous space can be summarized as follows: 1) apply the Reynolds decomposition to the velocity and pressure, i.e., $u_{n}=U_{n}+u_{n}^{\prime}$ and $p=P+p^{\prime}$ (where the capital letter and the prime denote the mean value and the fluctuation, respectively) and substitute them into Eq. (2); 2) subtract the transport equation for mean velocities; 3 ) multiply $2 u^{\prime}$; and 4) take the ensemble average. The resulting transport equation for RS reads

$$
\frac{\partial\left\langle u_{n}^{\prime} u_{m}^{\prime}\right\rangle}{\partial t}+C_{n m}=P_{n m}+T_{n m}+\Psi_{n m}+\Phi_{n m}+D_{n m}+\epsilon_{n m},
$$

where the angle bracket denotes the ensemble average. The first and second terms in the left-hand-side (LHS) are the unsteady term and the mean convection term $\left(C_{n m}\right)$, respectively. 
The right-hand-side (RHS) terms are the production $\left(P_{n m}\right)$, turbulent diffusion $\left(T_{n m}\right)$, pressure diffusion $\left(\Psi_{n m}\right)$, pressure strain $\left(\Phi_{n m}\right)$, viscous diffusion $\left(D_{n m}\right)$, the viscous dissipation $\left(\epsilon_{n m}\right)$, i.e.,

$$
\begin{aligned}
C_{n m} & =U_{k} \frac{\partial\left\langle u_{n}^{\prime} u_{m}^{\prime}\right\rangle}{\partial x_{k}}, \\
P_{n m} & =-\left\langle u_{m}^{\prime} u_{k}^{\prime}\right\rangle \frac{\partial U_{n}}{\partial x_{k}}-\left\langle u_{n}^{\prime} u_{k}^{\prime}\right\rangle \frac{\partial U_{m}}{\partial x_{k}}, \\
T_{n m} & =-\frac{\partial\left\langle u_{k}^{\prime} u_{m}^{\prime} u_{n}^{\prime}\right\rangle}{\partial x_{k}}, \\
\Psi_{n m} & =-\frac{\partial\left\langle u_{m}^{\prime} p^{\prime}\right\rangle}{\partial x_{n}}-\frac{\partial\left\langle u_{n}^{\prime} p^{\prime}\right\rangle}{\partial x_{m}}, \\
\Phi_{n m} & =\left\langle p^{\prime} \frac{\partial u_{n}^{\prime}}{\partial x_{m}}\right\rangle+\left\langle p^{\prime} \frac{\partial u_{m}^{\prime}}{\partial x_{n}}\right\rangle, \\
D_{n m} & =\frac{1}{\operatorname{Re}} \frac{\partial^{2}\left\langle u_{n}^{\prime} u_{m}^{\prime}\right\rangle}{\partial x_{k} \partial x_{k}}, \\
\epsilon_{n m} & =-\frac{2}{\operatorname{Re}}\left\langle\frac{\partial u_{n}^{\prime}}{\partial x_{m}} \frac{\partial u_{m}^{\prime}}{\partial x_{n}}\right\rangle,
\end{aligned}
$$

where Einstein's summation convention holds for the dummy index $k$.

The TKE is a sum of Reynolds normal stresses, i.e.,

$$
K=\frac{1}{2}\left(\left\langle u_{1}^{\prime} u_{1}^{\prime}\right\rangle+\left\langle u_{2}^{\prime} u_{2}^{\prime}\right\rangle+\left\langle u_{3}^{\prime} u_{3}^{\prime}\right\rangle\right)
$$

and the TKE budget reads

$$
\frac{\partial K}{\partial t}+C_{K}=P_{K}+T_{K}+\Psi_{K}+\Phi_{K}+D_{K}+\epsilon_{K}
$$

Each term in RHS is a half of the summation of the three normal components of the corresponding term in the RS budget, e.g., $P_{K}=\frac{1}{2} \sum_{n=1}^{3} P_{n n}$.

\section{Consistent scheme for Reynolds Stress and turbulent kinetic energy budgets}

\subsection{Discretization of Reynolds normal stress budget}

We define the Reynolds normal stress component $\left(\left\langle u_{n}^{\prime} u_{n}^{\prime}\right\rangle, n=1 \ldots 3\right)$ on each cell surface where the velocity component $u_{n}$ is defined. Hereafter, we omit the angle bracket of the ensemble average for notational simplicity.

The discretized terms composing the RS budget can be derived in the same manner as we derived the RS budget in the continuous space. First, $u_{n}^{\prime}$ is multiplied to the discretized $x_{n}$-momentum equation. The $u_{n}$-multiplied terms are then divided and/or merged to have the similar mathematical forms as those in the continuous space. At this step, the relevant interpolation schemes are used so that unwanted terms properly vanishes under the discretized continuity. The detailed derivation process is given in Appendix A, by taking the production and turbulent diffusion terms for examples, to which interested readers are referred.

As the result, the consistent discretization of RS budget can be expressed as

$$
\begin{aligned}
& C_{n n}={\overline{U_{k}}}^{x_{n}} x_{k} \frac{\delta{\widetilde{u_{n}^{\prime} u_{n}^{\prime}}}^{x_{k}}}{\delta x_{k}}, \\
& P_{n n}=-2 u_{n}^{\prime}{\overline{\bar{u}_{k}^{x_{n}}}}^{x_{k}} \frac{\delta{\overline{U_{n}}}^{x_{k}}}{\delta x_{k}}, \\
& T_{n n}=-\frac{\delta}{\delta x_{k}} \widetilde{u_{k}^{\prime}} \widetilde{x_{n}} \widetilde{u_{n}^{\prime} u_{n}^{\prime}} x_{k}, \\
& \Psi_{n n}=-2 \frac{\delta}{\delta x_{n}}{\overline{u_{n}^{\prime}}}^{x_{n}} p^{\prime}, \\
& \Phi_{n n}=2 p^{\prime} \frac{\delta u_{n}^{\prime}}{\delta x_{n}} x_{n},
\end{aligned}
$$




$$
\begin{aligned}
& D_{n n}=\frac{1}{\operatorname{Re}} \frac{\delta^{2} u_{n}^{\prime 2}}{\delta x_{k} \delta x_{k}}, \\
& \epsilon_{n n}=-\frac{2}{\operatorname{Re}} \frac{\delta u_{n}^{\prime}}{\delta x_{k}} \frac{\delta u_{n}^{\prime}}{\delta x_{k}},
\end{aligned}
$$

where the tilde and the hat denote the squared value and volume weighted average defined, e.g., as ${ }^{(4)}$

$$
\left.\frac{\delta}{\delta y} \widetilde{f^{\prime} g^{\prime}}\right|_{i+1 / 2, j, k}=\frac{1}{\Delta y_{j}}\left(f_{i+1 / 2, j+1, k}^{\prime} g_{i+1 / 2, j, k}^{\prime}-f_{i+1 / 2, j-1, k}^{\prime} g_{i+1 / 2, j, k}^{\prime}\right),
$$

and

$$
\left\{\begin{aligned}
\hat{u}_{i, j, k}^{x} & =\frac{\Delta x_{i+1 / 2} u_{i+1 / 2, j, k}+\Delta x_{i-1 / 2} u_{i-1 / 2, j, k}}{\Delta x_{i+1 / 2}+\Delta x_{i-1 / 2}}, \\
\hat{u}_{i+1 / 2, j+1 / 2, k}^{y} & =\frac{\Delta y_{j+1} u_{i+1 / 2, j+1, k}+\Delta y_{j} u_{i+1 / 2, j, k}}{\Delta y_{j+1}+\Delta y_{j}} .
\end{aligned}\right.
$$

The $f^{\prime}$ and $g^{\prime}$ are arbitrary values. It should be noted that the volume weighted average (27) is different from the volume-flux average (8): the former uses the weight of volume regardless of the directions of velocity and interpolation, while the latter does not.

The difference from the existing schemes is clear from comparison with formula presented in Appendix B. Note that the viscous diffusion and dissipation terms, Eqs. (24) and (25), are identical to those presented by Kajishima ${ }^{(21)}$.

\subsection{Discretization of turbulent kinetic energy budget}

The discretized TKE has conventionally ${ }^{(5),(6)}$ been defined by the arithmetic average at the center of the cell, i.e.,

$$
K=\frac{1}{2} \overline{u_{n}^{\prime} u_{n}^{\prime}} x_{n}
$$

where the summation convention holds for the dummy index $n$.

\subsection{Discretized of Reynolds shear stress budget}

We define the Reynolds shear stress (RSS, $n \neq m$ ) on the center of the cell. The discretized time derivative term is expressed as

$$
\left.\frac{\delta u_{n}^{\prime} u_{m}^{\prime}}{\delta t}\right|_{i, j}={\widehat{u_{m}^{\prime}}}^{x_{m}} \frac{{\widehat{\delta u_{n}^{\prime}}}_{\delta t}^{x_{n}}}{\left.\right|_{i, j}}+\left.{\widehat{u_{n}^{\prime}}}^{x_{n}} \frac{{\widehat{\delta u_{m}^{\prime}}}_{\delta t}^{x_{m}}}{\delta}\right|_{i, j}
$$

Here, the first and second terms in RHS represents the contributions from $u_{n}^{\prime}$ - and $u_{m}^{\prime}$ - momentum equations, respectively. The discretized RSS budget based on Eq. (29) can be expressed as

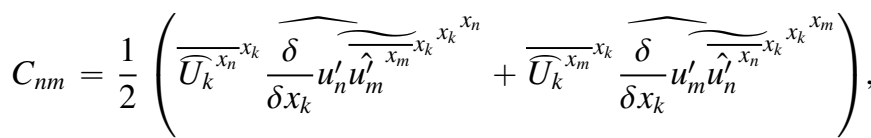

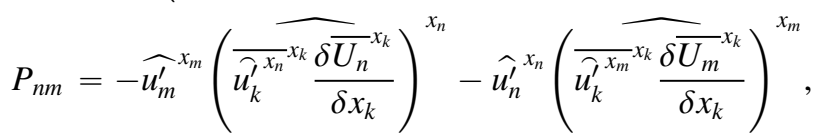

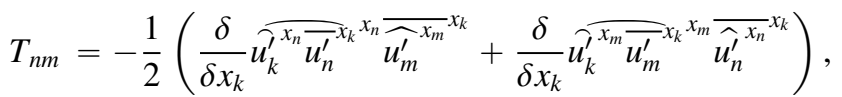

$$
\begin{aligned}
& \Psi_{n m}=-\frac{\delta}{\delta x_{n}}{\overline{\hat{u}_{m}^{\prime}}}^{x_{m}}{ }^{x_{n}}{\overline{p^{\prime}}}^{x_{n}}-\frac{\delta}{\delta x_{m}}{\overline{\hat{u}_{n}^{\prime}}}^{x_{n}} \bar{p}_{m}{\overline{p^{\prime}}}^{x_{m}}, \\
& \Phi_{n m}=\left({\overline{p^{\prime}}}^{x_{n} \frac{\widehat{\delta \hat{u}_{m}^{\prime}}}{\delta x_{n}}}\right)^{x_{n}}+\left({\overline{p^{\prime}}}^{x_{m} \frac{\delta{\widehat{u_{n}^{\prime}}}^{x_{n}}}{\delta x_{m}}}\right)^{x_{m}}
\end{aligned}
$$




$$
\begin{aligned}
& D_{n m}=\frac{1}{\operatorname{Re}}\left(\frac{\delta^{2}}{\delta x_{k} \delta x_{k}}{\widehat{u_{n}^{\prime}}}^{x_{n}}{\widehat{u_{m}^{\prime}}}^{x_{m}}\right),
\end{aligned}
$$

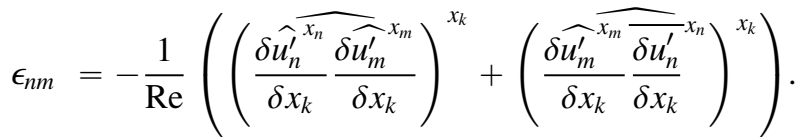

Because the energy-conservative FDM ensures the conservation of the TKE (i.e., $K$ ) but not of RSS commutation errors remain in convective, the turbulent diffusion, the viscous diffusion, and the viscous dissipation terms. While the different definitions of the RSS can be considered due to the staggered grid system, we found that with the other definitions induce the residual which is larger than that by the presented scheme.

\section{Numerical test}

Validity of the proposed scheme is examined in a fully development turbulent channel flow under a constant mass flow rate simulated by using the DNS code of Fukagata et al. ${ }^{(22)}$. The flow geometry is shown in Fig. 2. Periodic condition is employed for homogeneous ( $x$ and $z$ ) directions and no-slip boundary condition is employed at the walls. Uniform mesh is used in $x$ and $z$ direction, while nonuniform mesh is adopted in the wall-normal $(y)$ direction. The bulk Reynolds number is $\operatorname{Re}_{b}=5600$ based on the channel half-width and the twice bulk velocity, which corresponds to the friction Reynolds number of $\operatorname{Re}_{\tau} \approx 180$. The simulations are started from a fully developed channel flow. The RS and TKE budgets are computed by averaging over the homogeneous direction. The temporal average is taken in the period of $t^{+}=0$ to $t^{+} \approx 4000$ (about 4000 samples), where the subscript of + denotes the wall unit.

Figure 3 shows the profiles of different terms in the RS and TKE budgets computed by using the present scheme (top), Suzuki and Kawamura (SK) scheme ${ }^{(18)}$ (middle), and the intuitive scheme ${ }^{(18)}$ (bottom). SK scheme is the consistent scheme on the uniform and staggered grid system (see, Appendix B. 1). The intuitive scheme is a scheme with "intuitive" discretization (see, Appendix B. 2). The symbol represents the budgets computed using the spectral DNS data ${ }^{(20)}$. Due to this flow geometry, the convective term and the gradient of homogeneous directions in Eqs. (19)-(25) and Eqs. (30)-(36) vanish. As is clear from the figure, the budgets computed by the present scheme agrees well with those of the spectral method. The intuitive scheme results in non-negligible residual due to the inconsistency. The residual of the present and the SK schemes are found to be negligibly small in the Reynolds normal stress and the TKE budget (Figs.3(a)-(d)). Because the mesh expansion rate in the wall-normal direction used in the present DNS is small, the difference between these two results are small. Figure 3(e) shows the RSS budget. The budget computed by the present scheme are in reasonable agreement with that computed by the spectral method. The residual found to be slightly smaller than that by the SK and the intuitive schemes.

\section{Conclusions}

A numerical scheme for the computation of Reynolds stress budget which is consistent with the second-order energy-conservative finite difference method on the nonuniform and staggered grid system has been proposed. Validity of the proposed scheme has been confirmed in direct numerical simulation of a fully-developed channel flow: the residuals in the Reynolds normal stress and TKE budgets are found to be smaller than those computed by the intuitive schemes. The residual computed by the existing scheme, which is discretized on uniform grid system, is also small. We also apply the same idea to the discretization of Reynolds shear stress budget, although the consistency is imperfect, be derived the numerical test shows that the residual is slightly smaller than that computed by the other schemes.

\section{Appendix A: Derivation of consistent scheme}

In this Appendix, we show the detailed derivation process of the present consistent scheme. For brevity, we take the production and turbulent diffusion terms in RS budget for 
example. We also assume two-dimensional flow in $x-y$ plane for notational simplicity. Extension to three dimensional case is straightforward and the other terms can be derived in the similar manner. The Reynolds decomposition, $\left(u=U+u^{\prime}, v=v^{\prime}\right)$ is applied to the convective term of Eq. (6). By multiplying the twice fluctuation of streamwise velocity, $2 u_{i+1 / 2, j}^{\prime}$, reads

$$
\left[-2 u^{\prime} \frac{\delta \widehat{v}^{x} \bar{u}^{y}}{\delta y}\right]_{i+1 / 2, j}=\left[-2 u^{\prime} \frac{\delta \widehat{v}^{\prime x} \bar{U}^{y}}{\delta y}\right]_{i+1 / 2, j}+\left[-2 u^{\prime} \frac{\delta{\widehat{v^{\prime}}}^{x}{\overline{u^{\prime}}}^{y}}{\delta y}\right]_{i+1 / 2, j},
$$

where the index for stencil $(k)$ is omitted.

First, the production term is considered. The first term of RHS of Eq. (A.1) becomes

$$
\begin{aligned}
& {\left[-2 u^{\prime} \frac{\delta{\widehat{v^{\prime}}}^{x} \bar{U}^{y}}{\delta y}\right]_{i+1 / 2, j}=-2 u_{i+1 / 2, j}^{\prime} \frac{1}{\Delta y_{j}}\left(\left[{\widehat{v^{\prime}}}^{x} \bar{U}^{y}\right]_{i+1 / 2, j+1 / 2}-\left[{\widehat{v^{\prime}}}^{x} \bar{U}^{y}\right]_{i+1 / 2, j-1 / 2}\right),} \\
& =-\frac{2 u_{i+1 / 2, j}^{\prime}}{\Delta y_{j}}\left({\overline{\widehat{v}^{\prime}}}^{y}{ }_{i+1 / 2, j}^{y} \bar{U}_{i+1 / 2, j+1 / 2}^{y}-{\overline{\bar{v}^{\prime}}}_{i+1 / 2, j}^{y} \bar{U}_{i+1 / 2, j-1 / 2}^{y}\right) \\
& -\frac{2 u_{i+1 / 2, j}^{\prime}}{\Delta y_{j}}\left(\frac{1}{2}\left(\widehat{v}_{i+1 / 2, j+1 / 2}^{\prime}-\widehat{v}_{i+1 / 2, j-1 / 2}^{\prime} x\right) \bar{U}_{i+1 / 2, j+1 / 2}^{y}\right. \\
& \left.+\frac{1}{2}\left({\widehat{v^{\prime}}}_{i+1 / 2, j+1 / 2}^{x}-{\widehat{v^{\prime}}}_{i+1 / 2, j-1 / 2}^{x}\right) \bar{U}_{i+1 / 2, j-1 / 2}^{y}\right), \\
& =\left[-2 u^{\prime}{\overline{v^{\prime}}}^{y} \frac{\delta \bar{U}^{y}}{\delta y}\right]_{i+1 / 2, j}+\left[-2 u^{\prime} \overline{\bar{U}}^{y} \frac{\delta{\widehat{v^{\prime}}}^{x}}{\delta y}\right]_{i+1 / 2, j}, \\
& =\left[-2 u^{\prime}{\overline{v^{\prime}}}^{y} \frac{\delta \bar{U}^{y}}{\delta y}\right]_{i+1 / 2, j}+\left[-2 u^{\prime} U \frac{\delta{\widehat{v^{\prime}}}^{x}}{\delta y}\right]_{i+1 / 2, j} \\
& +O\left(\Delta y^{2}\right)
\end{aligned}
$$

where the identity equations, e.g.,

$$
\begin{aligned}
& {\widehat{v^{\prime}}}_{i+1 / 2, j+1 / 2}^{x}={\widehat{\widehat{v}^{\prime}}}_{i+1 / 2, j}^{y}+\frac{1}{2}\left({\widehat{v^{\prime}}}_{i+1 / 2, j+1 / 2}^{x}-{\widehat{v^{\prime}}}_{i+1 / 2, j-1 / 2}^{x}\right), \\
& {\widehat{v^{\prime}}}_{i+1 / 2, j-1 / 2}^{x}={\widehat{\widehat{v}^{\prime}}}_{i+1 / 2, j}^{y}+\frac{1}{2}\left({\widehat{v^{\prime}}}_{i+1 / 2, j-1 / 2}^{x}-{\widehat{v^{\prime}}}_{i+1 / 2, j+1 / 2}^{x}\right) .
\end{aligned}
$$

have been used. The first term of Eq. (A.2) is the production term. The second term vanishes due to the continuity in two cells sandwiching $i+1 / 2, j$ because a similar term (i.e., $\left.\left[-2 u^{\prime} U \delta{\widehat{u^{\prime}}}^{y} / \delta x\right]_{i+1 / 2, j}\right)$ is derived from the $x$-derivative component of the advection term.

Second, the turbulent diffusion term is considered. The second term of RHS of Eq. (A.1) becomes

$$
\begin{aligned}
& {\left[-2{u^{\prime}}^{\left.\frac{\delta{\widehat{v^{\prime}}}^{x}}{\delta u^{\prime}}\right]^{y}}\right]_{i+1 / 2, j}=-2 u_{i+1 / 2, j}^{\prime} \frac{1}{\Delta y_{j}}\left(\left[{\widehat{v^{\prime}}}^{x} \overline{u^{\prime}}\right]_{i+1 / 2, j+1 / 2}-\left[{\widehat{v^{\prime}}}^{x}{\overline{u^{\prime}}}^{y}\right]_{i+1 / 2, j-1 / 2}\right),} \\
& =-\frac{2}{\Delta y_{j}}\left(u_{i+1 / 2, j}^{\prime} \widehat{v}_{i+1 / 2, j+1 / 2}^{\prime}\left(\frac{u_{i+1 / 2, j+1}^{\prime}+u_{i+1 / 2, j}^{\prime}}{2}\right)\right. \\
& \left.-u_{i+1 / 2, j}^{\prime}{\widehat{v^{\prime}}}_{i+1 / 2, j-1 / 2}^{x}\left(\frac{u_{i+1 / 2, j}^{\prime}+u_{i+1 / 2, j-1}^{\prime}}{2}\right)\right) \text {, } \\
& =-\frac{1}{\Delta y_{j}}({\widehat{v^{\prime}}}_{i+1 / 2, j+1 / 2}^{x} \underbrace{u_{i+1}^{\prime}}_{{\widetilde{u^{\prime} u^{\prime}}}_{i+1 / 2, j+1 / 2}^{u_{i+1 / 2, j+1}^{\prime}} u_{i+1 / 2, j}^{\prime}} \\
& -{\widehat{v^{\prime}}}_{i+1 / 2, j-1 / 2}^{x} \underbrace{u_{i+1 / 2, j}^{\prime} u_{i+1 / 2, j-1}^{\prime}}_{{\widetilde{u^{\prime} u^{\prime}}}_{i+1 / 2, j-1 / 2}^{y^{\prime}}}) \\
& -\frac{1}{\Delta y_{j}} u_{i+1 / 2, j}^{\prime 2}\left({\widehat{v^{\prime}}}_{i+1 / 2, j+1 / 2}^{x}-{\widehat{v^{\prime}}}_{i+1 / 2, j-1 / 2}^{x}\right),
\end{aligned}
$$




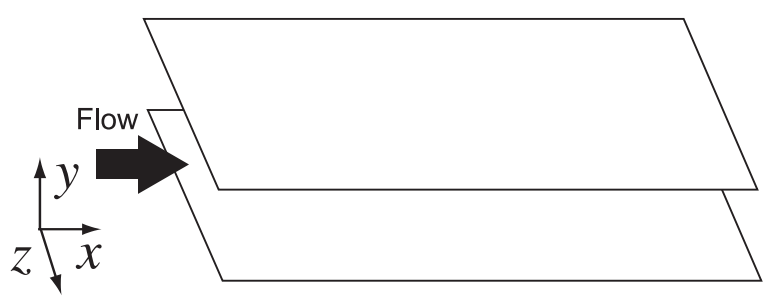

Fig. 2 Flow geometry

$$
=\left[-\frac{\delta{\widehat{v^{\prime}}}^{x} \widetilde{u^{\prime} u^{\prime}}}{\delta y}\right]_{i+1 / 2, j}+\left[-u^{\prime 2} \frac{\delta{\widehat{v^{\prime}}}^{x}}{\delta y}\right]_{i+1 / 2, j} .
$$

The first term of RHS is the turbulent diffusion term; the second term vanishes due to the continuity similarly to above.

\section{Appendix B: Existing schemes}

B.1. Suzuki and Kawamura scheme $e^{(18)}$

The consistent scheme proposed by Suzuki and Kawamura scheme ${ }^{(18)}$ reads

$$
\begin{aligned}
& P_{n n}=-2 u_{n}^{\prime} \overline{{\overline{u_{k}^{\prime}}}^{x_{n}} \frac{\delta U_{n}}{\delta x_{k}}}{ }^{x_{k}}, \\
& T_{n n}=\frac{\delta \overline{u_{n}^{\prime}} x_{n}{\overline{u_{n}^{\prime}}}_{n}^{x_{n}}{\overline{u_{k}^{\prime}}}^{x_{k}}}{\delta x_{k}}, \\
& \Psi_{n n}=-2 \frac{\delta \overline{\bar{u}_{n}^{\prime} p_{n}} p^{\prime}}{\delta x_{n}}, \\
& \Phi_{n n}=-2 p^{\prime} \overline{\delta u_{n}^{\prime}} \frac{x_{n}}{\delta x_{n}}, \\
& D_{n n}=\frac{1}{\operatorname{Re}} \frac{\delta^{2}\left(u_{n}^{\prime} u_{n}^{\prime}\right)}{\delta x_{k} \delta x_{k}}, \\
& \epsilon_{n n}=-\frac{2}{\operatorname{Re}} \frac{\delta u_{n}^{\prime}}{\delta x_{k}} \frac{\delta u_{n}^{\prime}}{\delta x_{k}} x_{k},
\end{aligned}
$$

where the RS is defined on the cell surface. Note that the turbulent diffusion term, Eq. (B.2), does not hold a consistency even on the uniform mesh. The TKE is computed by using arithmetic average at the center of the cell similarly to Eq. (28).

They also proposed the consistent scheme for RSS defined at the cell center reads

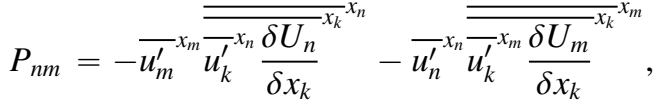

$$
\begin{aligned}
& T_{n m}=-\frac{\delta u_{k}^{\prime}{\overline{\overline{u_{n}^{\prime}}}}^{x_{n}} x_{k}{\overline{\overline{u_{m}^{\prime}}}}^{x_{m}} x_{k}}{\delta x_{k}}, \\
& \Psi_{n m}=-\frac{\delta{\overline{{\overline{u_{n}^{\prime}}}^{x_{n}}}}^{x_{m}}{\overline{p^{\prime}}}^{x_{m}}}{\delta x_{m}}-\frac{\delta{\overline{{\overline{u_{m}^{\prime}}}^{x_{m}}}}^{x_{n}}{\overline{p^{\prime}}}^{x_{n}}}{\delta x_{n}},
\end{aligned}
$$

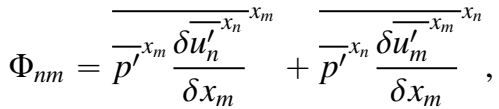

$$
\begin{aligned}
& D_{n m}=\frac{1}{\operatorname{Re}} \frac{\delta^{2}\left(\overline{u_{n}^{\prime}} x_{n} \overline{u_{m}^{\prime}} x_{m}\right)}{\delta x_{k} \delta x_{k}}, \\
& \epsilon_{n m}=-\frac{2}{\operatorname{Re}} \overline{\frac{\delta{\overline{u_{n}^{\prime}}}^{x_{n}}}{\delta x_{k}} \frac{\delta{\overline{u_{m}^{\prime}}}^{x_{m}}}{\delta x_{k}}} .
\end{aligned}
$$



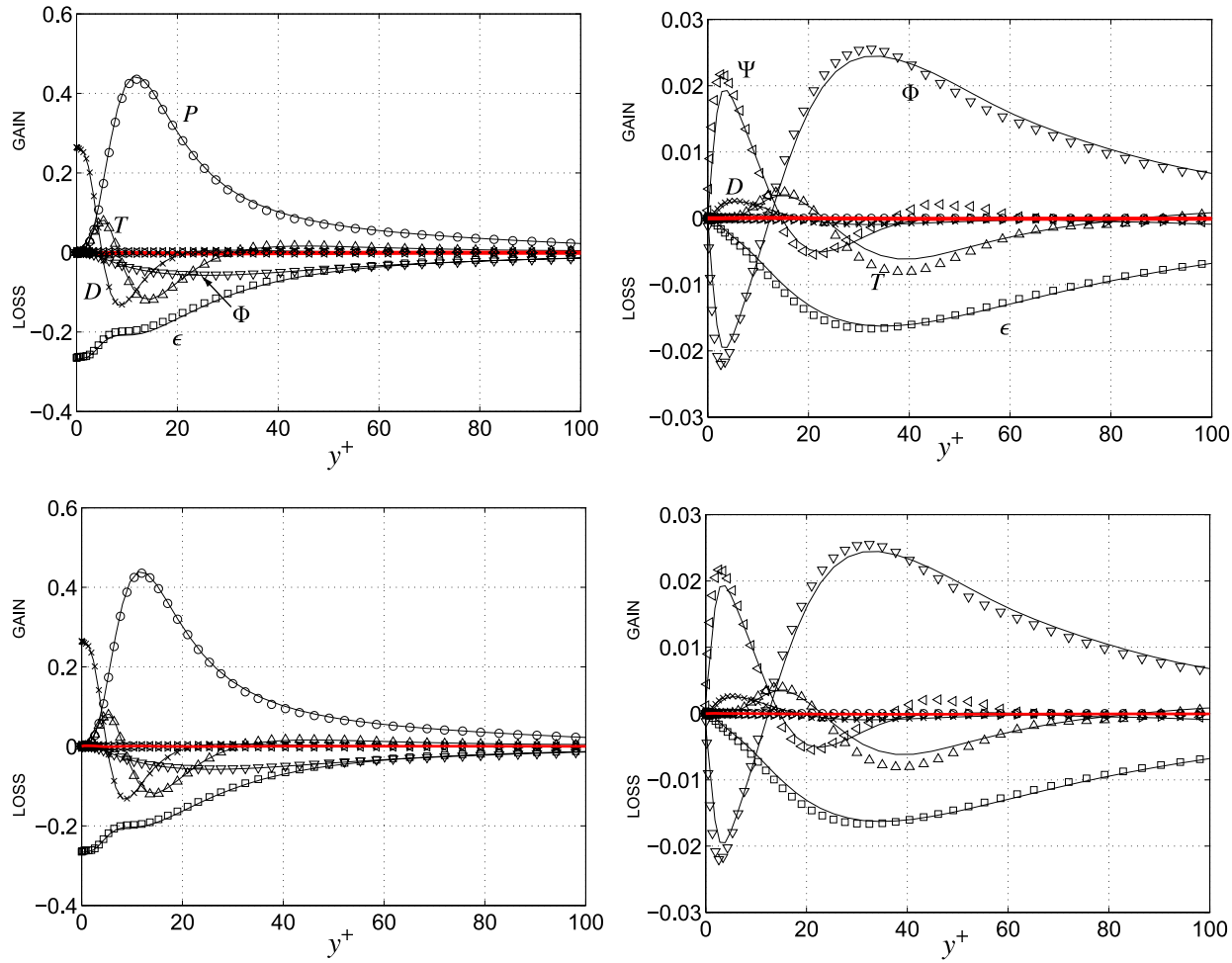

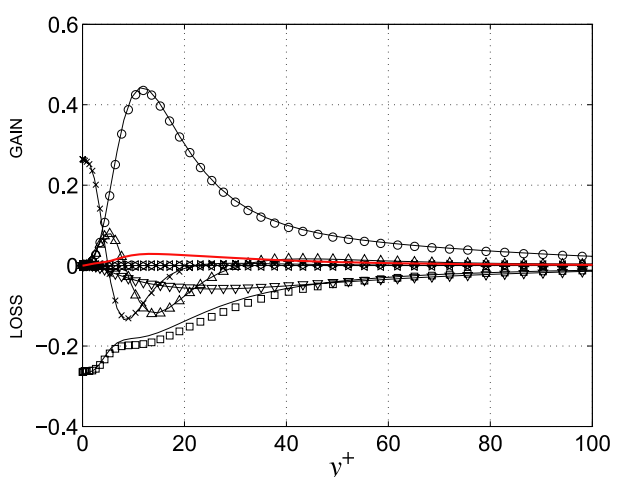

(a) $u^{\prime} u^{\prime}$

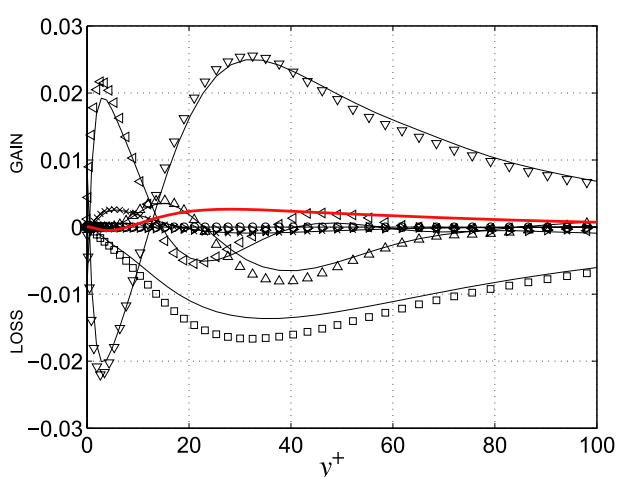

(b) $v^{\prime} v^{\prime}$

Fig. 3 RS and TKE budgets in fully developed channel flow at $\operatorname{Re}_{\tau} \approx 180: P$, production; $T$, turbulent diffusion; $\Psi$, pressure diffusion; $\Phi$, pressure strain; $D$, viscous diffusion; $\epsilon$, viscous dissipation. The solid lines represent: (top) the present scheme; (middle) Suzuki and Kawamura scheme ${ }^{(18)}$; and (bottom) the intuitive scheme ${ }^{(18)}$. The symbols represent the results by the spectral method ${ }^{(20)}$. The red thick lines and $\triangleright$ are the residuals computed by these schemes and the spectral method, respectively. 

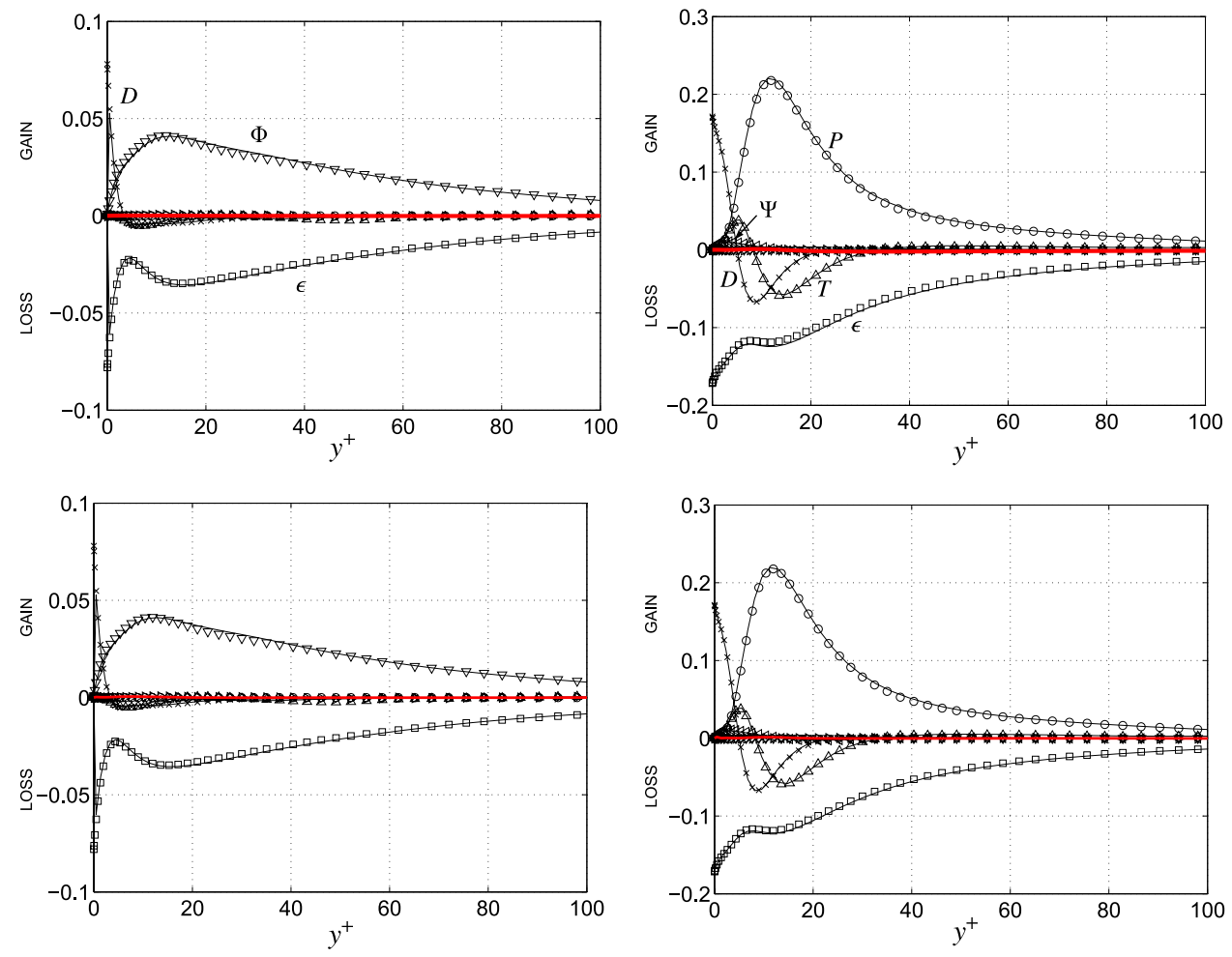

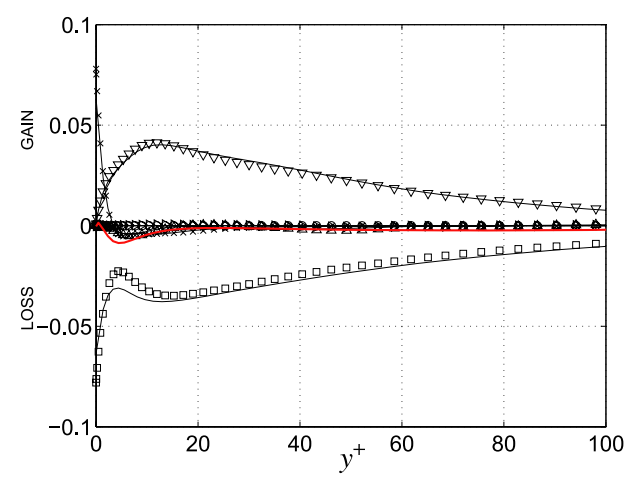

(c) $w^{\prime} w^{\prime}$.

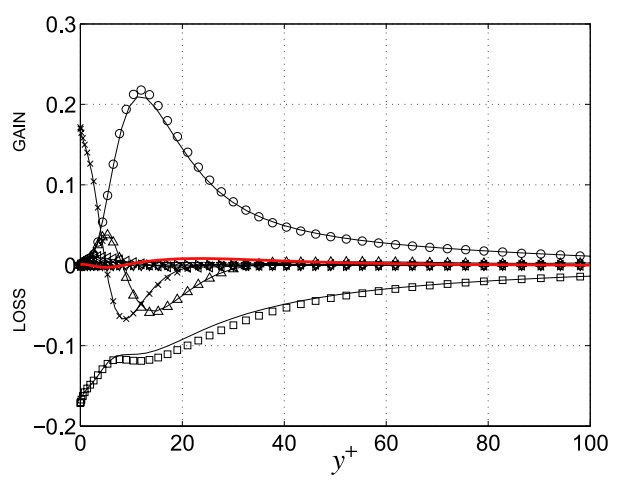

(d) TKE.

Fig. 3 Continued.

\section{B.2. Intuitive scheme}

The intuitive scheme ${ }^{(18)}$ reads

$$
\begin{aligned}
& P_{n m}=-{\overline{u_{n}^{\prime}}}^{x_{n}}{\overline{u_{k}^{\prime}}}^{x_{k}}{\overline{\frac{\delta U_{m}}{\delta x_{k}}}}^{x_{k}}-{\overline{u_{m}^{\prime}}}^{x_{m}}{\overline{u_{k}^{\prime}}}^{x_{k}}{\overline{\frac{\delta U_{n}}{\delta x_{k}}}}^{x_{k}}, \\
& T_{n m}=\frac{\overline{\delta \overline{u_{n}^{\prime}} x_{n} \overline{u_{m}^{\prime}} x_{m} \overline{u_{k}^{\prime}}}}{\delta x_{k}} x_{k}^{x_{k}}, \\
& \Pi_{n m}=-{\overline{u_{n}^{\prime}}}^{x_{n}} \frac{\Delta p^{\prime}}{\Delta x_{n}}-{\overline{u_{m}^{\prime}}}^{x_{m}} \frac{\Delta p^{\prime}}{\Delta x_{n}}, \\
& D_{n m}=\frac{1}{\operatorname{Re}} \frac{\delta^{2}\left(\overline{u_{n}^{\prime}} x_{n} \overline{u_{m}^{\prime}} x_{m}\right)}{\delta x_{k} \delta x_{k}}, \\
& \epsilon_{n m}=-\frac{2}{\operatorname{Re}}\left(\overline{\frac{\Delta u_{n}^{\prime}}{\Delta x_{k}}} \frac{x_{n}}{\frac{\Delta u_{m}^{\prime}}{\Delta x_{k}}}\right),
\end{aligned}
$$



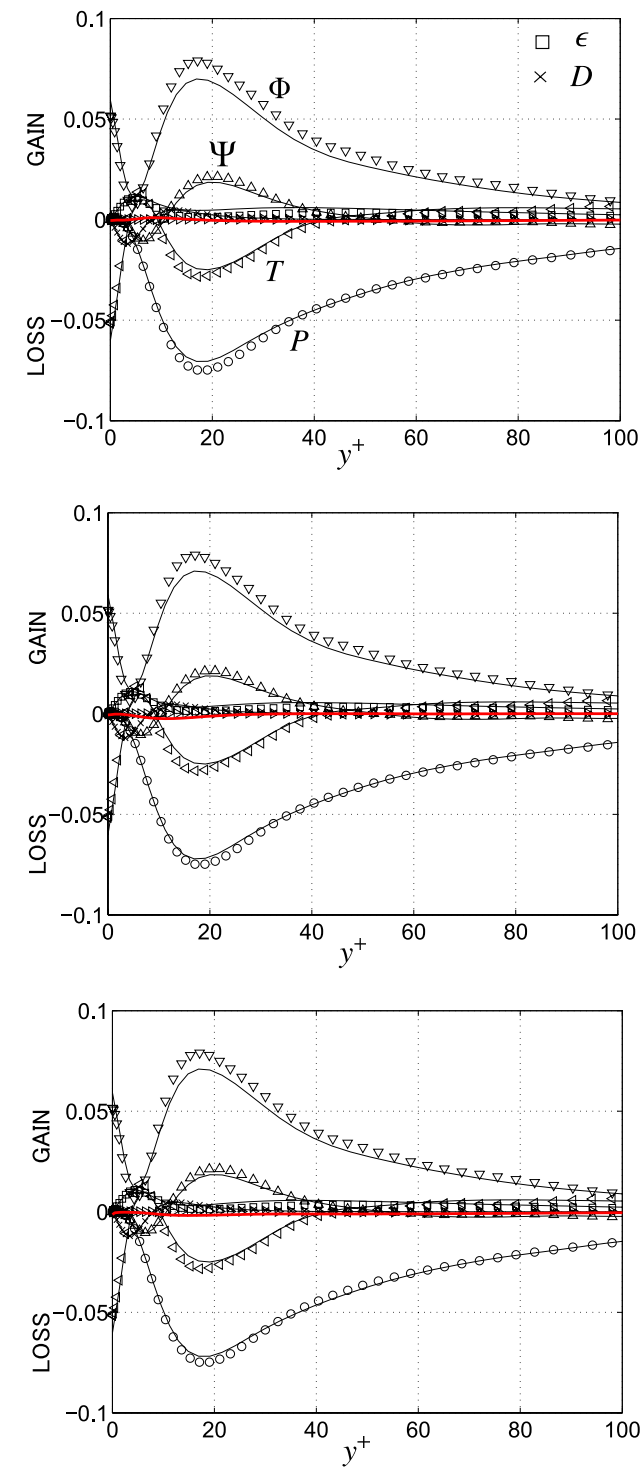

(e) $u^{\prime} v^{\prime}$

Fig. 3 Continued.

where the differential operator is defined, e.g., as

$$
\left[\frac{\Delta p^{\prime}}{\Delta x}\right]_{i, j, k}=\frac{p_{i+1, j, k}^{\prime}-p_{i-1, j, k}^{\prime}}{2 \Delta x_{i}},
$$

and the RS is defined at the cell center. Because they presented the pressure-oriented terms altogether as the velocity-pressure gradient term, $\Pi_{n n}$, we instead used Eq. (22) and (23) for computation of the pressure diffusion and the pressure strain terms of Fig. 3. The TKE is simply defined as the summation of Reynolds normal stresses defined at the center of the cell.

\section{Acknowledgments}

The authors are grateful to Dr. Shinnosuke Obi (Keio University). This work was supported through Keio University Global COE program "Center for Education and Research of Symbiotic, Safe and Secure System Design".

\section{References}

( 1 ) Orszag, S. A. and Patterson, G. S., Numerical simulation of three-dimensional homogeneous isotropic turbulence, Phys. Rev. Lett., Vol. 28 (1972), pp. 76-79. 
( 2 ) Kim, J., Moin, P., and Moser, R., Turbulence statistics in fully development channel flow at low Reynolds number, J. Fluids Mech., Vol. 177 (1987), pp. 133-166.

( 3 ) Harlow, F. H. and Welch, J. E., Numerical calculation of time-dependent viscous incompressible flow of fluid with free surface, Phys. Fluids, Vol. 8 (1965), pp. 2182-2189.

( 4 ) Piacsek, S. A. and Williams, G. P., Conservation properties of convection difference sheme, J. Comput. Phys., Vol. 6 (1970), pp. 392-405.

( 5 ) Morinishi, Y., Lund, T. S., Vasilyev, O. V., and Moin, P., Fully conservative higher order finite difference scheme for incompressible flow, J. Comput. Phys., Vol. 143 (1998), pp. 90-124.

( 6 ) Ham, F. E., Lien, F. S., and Strong, A. B., A fully conservative second-order finite difference scheme for incompressible flow on nonuniform grids, J. Comput. Phys., Vol. 177 (2002), pp. 117-133.

( 7 ) Kajishima, T., Finite-difference method for convective terms using non-uniform grid, Trans. JSME/B, Vol. 65, No. 633 (1999), pp. 1607-1612 (in Japanese).

( 8 ) Bewley, T. R., Optimal and robust control and estimation of transition, convection, and turbulence, Doctral thesis, Stanford University (1999).

(9) Fukagata, K. and Kasagi, N., Highly energy-conservative finite difference method for the cylindrical coordinate system, J. Comput. Phys., Vol. 181 (2002), pp. 478-498.

(10) Morinishi, Y., Vasilyev, O. V., and Ogi, T., Fully conservative finite difference scheme in cylindrical coordinates for incompressible flow simulations, J. Comput. Phys., Vol. 197 (2004), pp. 686-710.

(11) Nikitin, N., Finite-difference method for incompressible Navier-Stokes equations in arbitrary orthogonal curvilinear coordinates, J. Comput. Phys., Vol. 217 (2006), pp. 759791.

(12) Desjardins, O., Blanquart, G., Balarac, G., and Pitsch, H., High order conservative finite difference scheme for variable density low Mach number turbulent flows, J. Comput. Phys., Vol. 227 (2008), pp. 7125-7159.

(13) Mansour, N. N., Kim, J., and Moin, P., Reynolds-stress and dissipation-rate budgets in a turbulent channel flow, J. Fluids Mech., Vol. 194 (1988), pp. 15-44.

(14) Launder, B. E., Reece, G. J., and Rodi, W., Progress in the development of a Reynolds stress turbulence closure, J. Fluid Mech., Vol. 68 (1975), pp. 537-566.

(15) Cambon, C. and Scott, J. F., Linear and nonlinear models of anisotropic turbulence, Annu. Rev. Fluid Mech., Vol. 31 (1999), pp. 1-53.

(16) Fukagata, K. and Kasagi, N., Drag reduction in turbulent pipe flow with feedback control applied partially to wall, Int. J. Heat Fluid Flow, Vol. 24 (2003), pp. 480-490.

(17) Kasagi, N., Suzuki, Y., and Fukagata, K., Microelectromechanical system-based feedback control of turbulence for skin friction reduction, Annu. Rev. Fluid Mech., Vol. 41 (2009), pp. 231-251.

(18) Suzuki, T. and Kawamura, H., Consistency of finite-difference scheme in direct numerical simulation of turbulence, Trans. JSME/B, Vol. 60, No. 578 (1994), pp. 3280-3286 (in Japanese).

(19) Kawamura, H, Direct numerical simulation of turbulence by finite difference scheme, The Recent Developments in Turbulence Research, (Int. Academic Publishers, 1995), pp. 54-60.

(20) Moser, R. D., Kim, J., and Mansour, N. N., Direct numerical simulation of turbulent channel flow up to $\mathrm{Re}_{\tau}=590$, Phys. Fluids, Vol. 11 (1999), pp. 943-945.

(21) Kajishima, T., Numerical Simulation of Turbulent Flows (Yokendo, Tokyo, 1999) (in Japanese), p. 89.

(22) Fukagata, K., Kasagi, N., and Koumoutsakos, P., A theoretical prediction of friction drag reduction in turbulent flow by superhydrophobic surfaces, Phys. Fluids, Vol. 18 (2006), 051703 . 